\title{
Wave Concept Iterative Procedure Analysis of Patch Antennas on Nanostructured Ceramic Substrates
}

\author{
V. P. Silva Neto ${ }^{*}$, C. F. L. Vasconcelos, M. R. M. L. Albuquerque, and A. G. D’Assunção \\ Universidade Federal do Rio Grande do Norte, 59072-970, Natal, RN, Brazil \\ " corresponding author, E-mail: valdemir. neto@yahoo.com.br
}

\begin{abstract}
The wave concept iterative procedure (WCIP) is proposed to analyze rectangular and circular patch antennas on nanostructured ceramic substrates. Principles of WCIP are described and advantages are emphasized. The analysis of microstrip antennas on double layered substrates is performed in space and spectral domains. In addition, Fast Fourier Transformation (FFT) is used to improve the efficiency of the method. WCIP simulated results are compared to HFSS software ones. A good agreement is observed.
\end{abstract}

\section{Introduction}

The wave concept iterative procedure (WCIP) is developed to analyze microstrip antennas on ceramic substrates. WCIP method, which uses the wave formulation for solving electromagnetic problems, is suitable for analysis of planar microwave circuit, as well as patch antennas [1-3]. This method is based upon a combination of tangential field and initial conditions to characterize the waves in a microwave circuit surface. Moreover, the WICP method uses FFT to reduce the required computing time and provide both versatility and reliable representation of the circuit structure.

In current communication systems, one of the processes which have attracted most of the researchers is the miniaturization of microwave circuits. The miniaturization of electronics systems requires small antennas. Nevertheless, a very good characterization of the antennas is needed. One of the available techniques to minimize the antenna size is the use of ceramic materials with very high permittivity [4-6]. One of the advantages of ceramic materials over other dielectric materials is the possibility of fabricating compact and easy to build several microwave integrated circuits, such as microstrip patch antennas [7].

Nanomaterials are being identified worldwide as the key for the discovery of a new generation of devices with many different revolutionary properties and functionalities. Also dielectric ceramics are materials that are being considered for the miniaturization of microwave devices, antennas, and equipments [5].
This paper proposes to analyze microstrip patch antennas on nanostructured dielectric layers by a wave iterative method. The finite elements simulation by HFSS is used to compare with WCIP results to validate the theoretical formulation.

\section{WCIP Formulation}

The wave concept iterative procedure is an integral method that use a full wave formulation based on concept of transversal waves to solve the electromagnetic problem. The principle of the WCIP is to describe the incident $\overrightarrow{\mathrm{A}}$ and reflected $\vec{B}$ waves at the boundaries interfaces as a function of the tangential electric and transverse electric current densities (or magnetic fields), as shown in Fig. 1.

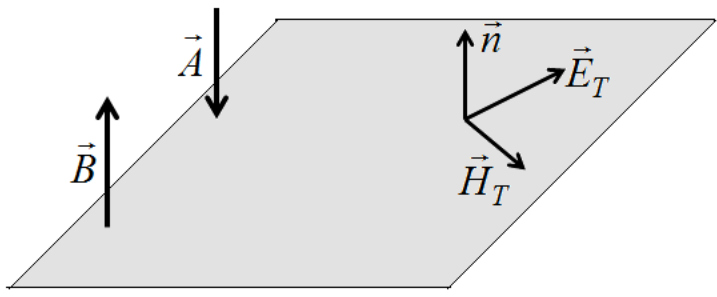

Figure 1: Incident and reflected waves on arbitrary surface.

The relationship between these incident and reflected waves and electromagnetic field is given by:

$$
\begin{gathered}
\overrightarrow{\mathrm{A}}=\frac{1}{2 \sqrt{\mathrm{Z}_{0}}}\left(\overrightarrow{\mathrm{E}}_{\mathrm{T}}+\mathrm{Z}_{0} \overrightarrow{\mathrm{J}}_{\mathrm{T}}\right) \\
\overrightarrow{\mathrm{B}}=\frac{1}{2 \sqrt{\mathrm{Z}_{0}}}\left(\overrightarrow{\mathrm{E}}_{\mathrm{T}}-\mathrm{Z}_{0} \overrightarrow{\mathrm{J}}_{\mathrm{T}}\right)
\end{gathered}
$$

where $Z_{0}$ is the intrinsic impedance of the medium given in (3), $\overrightarrow{\mathrm{E}}_{\mathrm{T}}$ is the electric field component that is tangential to the surface and $\vec{n}$ is the normal vector to the surface. The transverse current density is expressed in terms of tangential magnetic field as defined in (4)

$$
\mathrm{Z}_{0}=\sqrt{\frac{\mu_{0}}{\varepsilon_{0} \varepsilon_{\mathrm{r}}}}
$$




$$
\overrightarrow{\mathrm{J}}_{\mathrm{T}}=\overrightarrow{\mathrm{H}}_{\mathrm{T}}^{\wedge} \overrightarrow{\mathrm{n}}
$$

Two operators are applied to such incident and reflected waves, generating the equations system (5) and (6). The solution of these equations enables to solve the electromagnetic problem.

$$
\begin{aligned}
\overrightarrow{\mathrm{A}} & =\hat{\mathrm{S}} \overrightarrow{\mathrm{B}}+\overrightarrow{\mathrm{A}}_{0} \\
\overrightarrow{\mathrm{B}} & =\hat{\Gamma} \overrightarrow{\mathrm{A}}
\end{aligned}
$$

The scattering operator $\hat{\mathrm{S}}$ takes into account the boundary conditions in the space domain that are the continuity of tangential electric fields. We have that the sum of the surface current density is null for a dielectric surface, and the sum of the tangential component of the electric field is null for a metallic surface. This means that for a metallic surface $S=-1$, and for a dielectric surface $S=1[6]$.

The reflection operator $\hat{\Gamma}$ takes into account the medium reaction, in the spectral domain. The elements of $\hat{\Gamma}$ are related to the propagation modes.

The multilayer structure is performed in the spectral domain. We use transmission line theory to calculate the impedance seen from the interface for each mode, instead of using the classical modal impedance of a homogeneous media. The new modal impedance is deduced for the cascade of each layer, as defined in [7].

\section{Nanostructured Substrates}

Nanostructured dielectric ceramics with high permittivity are commonly applied in several microwave communication circuits. Once high-permittivity dielectrics can be used as substrates for antennas, with advantages of compact size and low profile, the development of ceramic substrate antennas for wireless communication systems is presently receiving a growing interest.

Over the years several papers have been dedicated to the study of microwave circuits on ceramic substrates, and to describe the experimental procedure to obtain this material. Generally, nanostructured ceramic substrates are obtained by traditional solid-state method. Chemical techniques, such as combustion and sol-gel, are used to produce these oxides with several advantages, namely, nanosized particles, high reactivity, and very good homogeneity in composition [8-9].

The purpose of this paper is to characterize microstrip patch antennas on ceramic substrates by means of an integral method and to compare these results with finite elements simulations. Here, the ceramic substrate was obtained at UFRN laboratory, by the experimental method described in [9].

\section{Results and Discussions}

Figure 2 shows de first planar circuit considered in this work. This structure consists in a rectangular patch antenna on two dielectric layers. The patch has width $\mathrm{W}=26.12 \mathrm{~mm}$ and length $\mathrm{L}=35.11 \mathrm{~mm}$. The antenna substrate is composed by two isotropic layers. Region 1 is characterized by $\mathrm{h}_{1}=1.57 \mathrm{~mm}$, relative electric permittivity $\varepsilon_{\mathrm{r} 1}$, and magnetic permeability $\mu_{0}$. Region 2 is characterized by $h_{2}=$ $1.57 \mathrm{~mm}$, relative electric permittivity $\varepsilon_{\mathrm{r} 2}$, and magnetic permeability $\mu_{0}$.
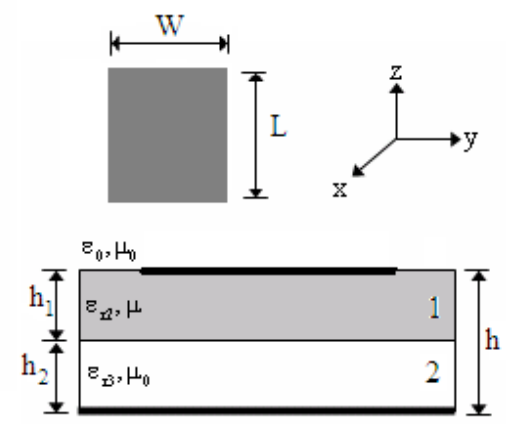

Figure 2: Rectangular patch antenna on two dielectric layers.

Figure 3 depicts the results of return loss for microstrip rectangular patch antenna on double layered substrate. Region 1 (Fig. 2) is a nanostructured ceramic layer $\left(\varepsilon_{\mathrm{r} 1}=20\right)$, and region 2 is a FR4 layer $\left(\varepsilon_{\mathrm{r} 1}=4.4\right)$. Simulations through Ansoft HFSS show that the antenna resonates at $2 \mathrm{GHz}$ with insertion loss equal to $-15 \mathrm{~dB}$, approximately. Results using WCIP indicate that the first resonant frequency is $2.1 \mathrm{GHz}$ and $\mathrm{S}_{11}=-7.8 \mathrm{~dB}$. In this case, the relative error on resonant frequency is $4.7 \%$, so a good agreement is verified from these results.

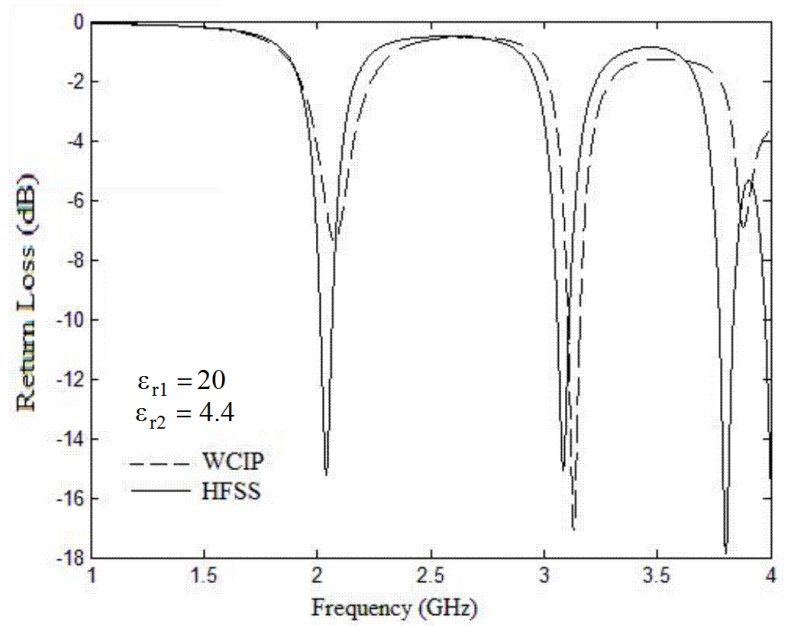

Figure 3: Return loss versus frequency for rectangular patch antenna.

In Fig. 4, the results are obtained for the rectangular patch printed on FR4 (region 1), and separated from the antenna ground plane by an air-filled layer (region 2). The antenna has resonant frequency at $3.6 \mathrm{GHz}$ with a bandwidth equal to $15 \mathrm{MHz}$, using the Ansoft HFSS simulation. The WCIP analysis indicates that the resonant frequency is $3.7 \mathrm{GHz}$ and $18 \mathrm{MHz}$ for the bandwidth is achieved. 


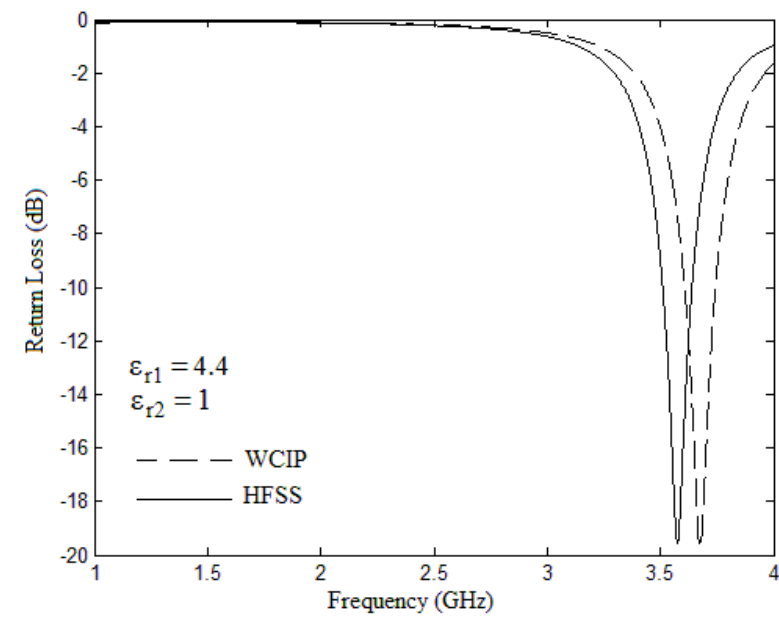

Figure 4: Return loss versus frequency for suspended microstrip rectangular patch antenna.

Figure 5 indicates the frequency responses for the considered antenna printed on suspended ceramic substrate. In this case, the antenna has resonant frequency at $3.1 \mathrm{GHz}$, by HFSS simulation. The WCIP simulation indicates the resonant frequency at $3.2 \mathrm{GHz}$, which implies an error of $3.2 \%$, if we compare this result with HFSS simulation.

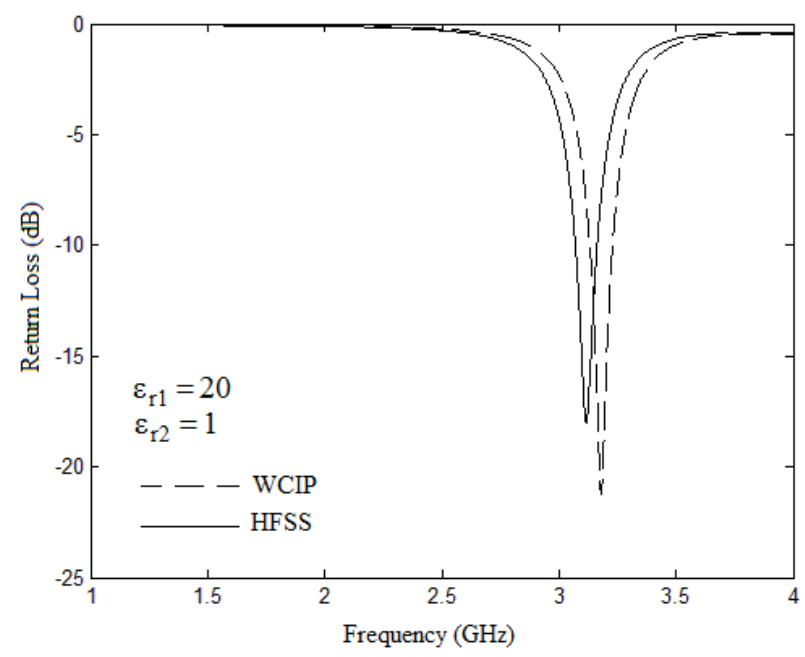

Figure 5: Return loss versus frequency for rectangular patch antenna on suspended nanostructured substrate.

Numerical results for circular patch antenna (Fig. 6) on two dielectric layers are presented in Figs. 7-9. The circular patch, with radius $\mathrm{R}=29 \mathrm{~mm}$, is supported by two isotropic layers. Region 1 has thickness $h_{1}=1.57 \mathrm{~mm}$, relative electric permittivity $\varepsilon_{\mathrm{rl}}$, and magnetic permeability $\mu_{0}$. Region 2 has $\mathrm{h}_{2}=1.57 \mathrm{~mm}$, relative electric permittivity $\varepsilon_{\mathrm{r} 2}$, and magnetic permeability $\mu_{0}$. The resonance of TM modes are analyzed by WCIP model and the results are compared with those obtained by Ansoft HFSS simulation to validate the theoretical formulation proposed.
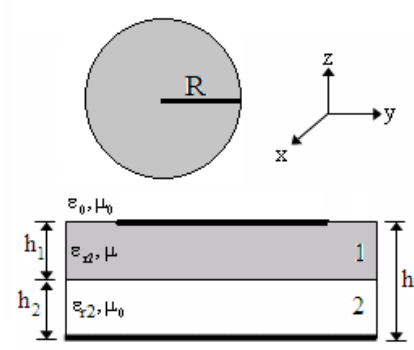

Figure 6: Circular patch antenna on two dielectric layers.

Figure 7 presents the frequency response of circular patch antenna on double layer substrate. The top layer is a nanostructured ceramic material $\left(\varepsilon_{\mathrm{r} 1}=20\right)$, and region 2 is a FR4 layer $\left(\varepsilon_{\mathrm{r} 1}=4.4\right)$. The antenna resonates (mode $\left.\mathrm{TM}_{11}\right)$ at $2.5 \mathrm{GHz}$, considering WCIP model and HFSS simulation, so this allows to conclude the validation of the proposed model.

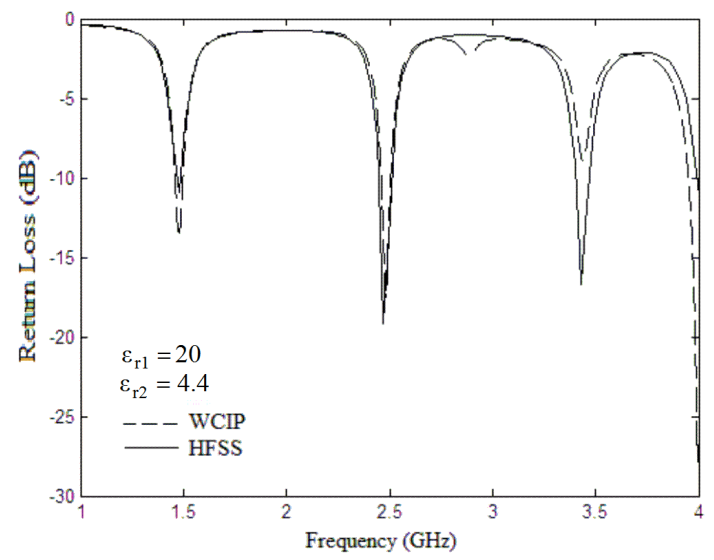

Figure 7: Return loss versus frequency for circular patch antenna.

Figure 8 depicts the insertion loss of the circular antenna illustrated in Fig. 6. The HFSS simulation indicates that the antenna has resonant frequency at $2.15 \mathrm{GHz}$, with return loss $\mathrm{S}_{11}=-22 \mathrm{~dB}$. The WCIP analysis indicates that the resonant frequency is $2.1 \mathrm{GHz}$, showing a relative error of $2.32 \%$.

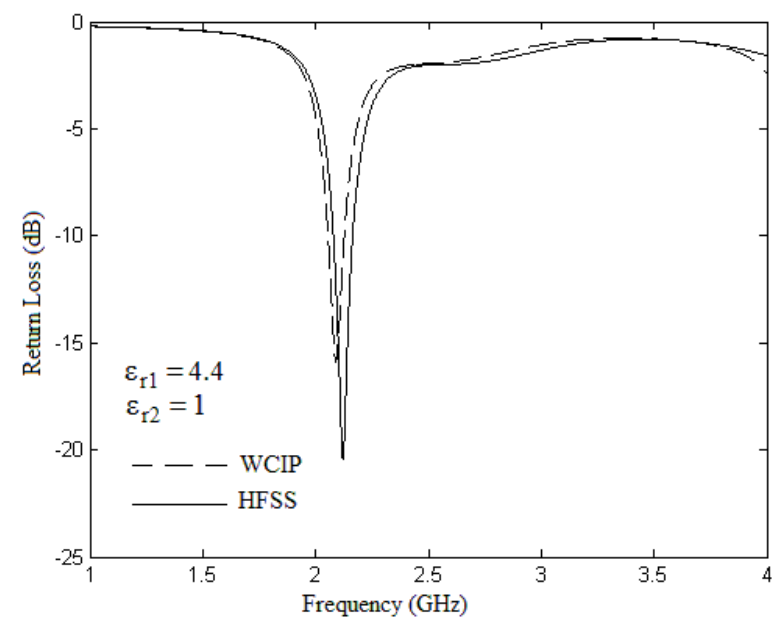

Figure 8: Return loss versus frequency for suspended microstrip circular patch antenna. 
The input return loss for the suspended circular patch antenna on ceramic substrate is shown in Fig. 9. The first resonant frequency of the antenna is chosen at $1.8 \mathrm{GHz}$, with $\mathrm{S}_{11}=-16 \mathrm{~dB}$, obtained by WCIP simulation. Results from the Ansoft HFSS indicate no good performance of the antenna at $1.8 \mathrm{GHz}$, because the return loss is, approximately, equal to $-6 \mathrm{~dB}$. However, the third resonant mode indicates a good agreement between WCIP simulation and HFSS results. From WCIP analysis, we obtain a resonant frequency at $3.7 \mathrm{GHz}$ with a level of insertion loss of $-17 \mathrm{~dB}$. Ansoft simulation indicates resonant frequency at $3.8 \mathrm{GHz}$ and return loss $\mathrm{S}_{11}=-19 \mathrm{~dB}$.

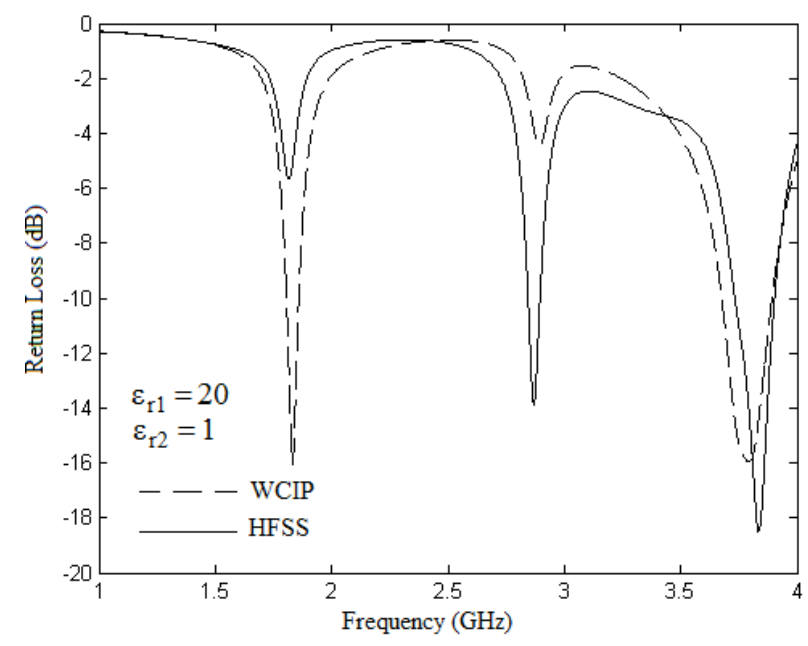

Figure 9: Return loss versus frequency for circular patch antenna on nanostructured suspended substrate.

\section{Conclusion}

An efficient method for the analysis of microstrip patch antennas was presented. The results demonstrated a very good agreement between HFSS and WCIP simulations, illustrating the accuracy of the proposed WCIP method. Furthermore, the use of a FFT algorithm has improved the required computing time. Also, this iterative method provides faster convergence as compared to integral methods, such as the method of moments, and the finite element method, because there is no need of matrix inversion and of large amount of memory space

The use of ceramic substrate allows reduce the physical dimensions of antenna. The analysis of other microwave circuits on ceramic substrates by WCIP formulation will be considered in future works.

\section{Acknowledgements}

We thank the Brazilian Research Agencies CNPq, CAPES and FAPERN for partial financial support.

\section{References}

[1] R. S. N'Gongo and H. Baudrand, "Application of Wave Concept Iterative Procedure in planar circuits," Recent Res. Devel. Microwave Theory and Technique, vol. 1, pp. 187-197, 1999.
[2] M. Titaouine, A. Gomes Neto, H. Baudrand, and F. Djahli, "Analysis of frequency selective surface on isotropic/anisotropic layer using WCIP method", ETRI Journal, vol.29, no. 1, pp.36 - 44, 2007.

[3] H. Meganafi, N. Boruklin-Hacene, H. Baudrand, N. Raveu, and I. Allam, "A Monolayer passive structure analysis by WCIP method", Sciences of Electronics, Technologies of Informatique and Telecomunications, SETIT-IEEE, 2012.

[4] F. S. Chute and G. B. Walker, "Suitability of Ni-Zn ferrites ceramics with controlled porosity as granular substrates for mobile handset miniaturized antennas", IEEE Transactions on Magnetics, vol. 47, n. 10, pp. 3720-3723, 2011.

[5] L. D. Jadhav, S. P. Patil, A. U. Chavan, A. P. Jamale, V. R. Puri, "Solution combustion synthesis of $\mathrm{Cu}$ nanoparticles: a role of oxidant-to-fuel ratio", IET Micro \& Nano Letters, pp. 812-815, 2011.

[6] N'Gongo S. Rufin, "Electromagnetic modeling of planar circuits, applied to the influence of box (in French)", PhD Tesis, ENSEEIHT, Toulouse, 1999.

[7] N. Raveu, G. Prigent, T. Callegari, H. Baudrand, "WCIP applied to active plasma circuits", Progress In Electromagnetic Research Letters, vol. 21, pp. 89 - 98, 2011.

[8] E.E.C. Oliveira, A.G.D’Assunção, J.B.L. Oliveira, A.M. Cabral, "Small size rectangular DRA antenna dual-band based in calcium titanate", Microwave and Optical Technology Letters, vol. 54, pp.976-979, 2012.

[9] R. Carneiro Filho, J. H. Araújo, M. F. Ginani, A. G. d'Assunção Jr, R. A. Martins, A. G. d'Assunção, L. M. Mendonça, "Simulation and measurement of inset-fed microstrip patch antennas on $\mathrm{BiNbO}_{4}$ substrates", Microwave and Optical Technology Letters, vol. 52, pp.1034-1036, 2010. 